\title{
Suppression of visible persistence in apparent motion
}

\author{
JOHN H. HOGBEN \\ University of Western Australia, Nedlands, Western Australia, Australia \\ and \\ VINCENT DI LOLLO \\ University of Alberta, Edmonton, Alberta, Canada
}

\begin{abstract}
Moving stimuli produce less smear than would be expected on the basis of visible persistence lasting 100-150 msec. Two experiments examined the duration of smear as a function of background luminance, target velocity, and duration of the display. It was found that smear decreased as background luminance increased, smear increased with velocity, and, as display duration increased from 10 to $160 \mathrm{msec}$, duration of smear first increased and then decreased. Alternative explanations of the results are considered. It is suggested that smear is actively suppressed by stimulus-initiated inhibitory processes.
\end{abstract}

There is abundant evidence that a brief display remains visible for about $120 \mathrm{msec}$ after its onset (e.g., Coltheart, 1980). This phenomenon, known as "visible persistence," creates a problem for the perception of moving objects. As the image sweeps across the retina, it stimulates a series of successive locations. Were visible persistence to last $120 \mathrm{msec}$ at each location, the moving object would be seen as trailing a wake of smear. The problem is that, under a wide range of viewing conditions, we see sharply defined objects moving against equally sharp backgrounds.

How does the visual system avoid smear? Eye movements fail to provide an adequate explanation. When a moving object is pursued, the background remains unsmeared; similarly, objects moving in different directions are seen without smear.

Burr $(1980,1981)$ has proposed an explanation in terms of two systems. One is tuned to low spatial and high temporal frequencies and is specialized to detect and analyze targets in motion. The other is tuned to high spatial and low temporal frequencies and responds optimally to stationary targets. Motion smear results from inappropriate activation of the latter system by a moving stimulus. Smear is avoided as soon as the motion-specific system is engaged.

An alternative, and perhaps complementary, account has been suggested by Di Lollo and Hogben (1985) in terms of active suppression of the visible persistence of a stimulus when another is presented nearby and soon af-

This research was supported by a study leave grant from the University of Western Australia to John H. Hogben, and by Natural Sciences and Engineering Research Council of Canada Grant A6592 to Vincent Di Lollo, whose mailing address is: Department of Psychology, University of Alberta, Edmonton, Alberta T6G 2E9, Canada. ter. We have argued elsewhere (Hogben \& Di Lollo, 1984) that the suppression could arise from inhibitory interactions between successively stimulated points, as in apparent motion and metacontrast.

It is the purpose of the present work to pursue the relationship between perceptual suppression of motion smear and inhibitory activity in the visual system. If inhibition does underlie the suppression of smear, then the degree of suppression should increase under conditions that favor inhibitory activity, notably, high levels of luminous intensity (Barlow, 1958).

In the present work, perceptual suppression was studied using Burr's (1980) technique of matching the length of a standard line with the perceived length of the smear produced by a point in apparent motion. However, before examining the effects of luminance on suppression, it was necessary to replicate Burr's (1980) findings. This was done in Experiment 1.

\section{EXPERIMENT 1}

Over a range of velocities, Burr (1980) found that amount of smear increased for durations of the display up to about $30 \mathrm{msec}$. It then decreased at longer durations as the impression of motion became more pronounced. The purpose of Experiment 1 was to replicate these results under display conditions that were virtually identical to Burr's. It must be noted at the outset that the stimuli employed in both Burr's (1980) and the present studies were in apparent-or stroboscopicmotion, rather than in real continuous motion. Therefore, the outcomes of these studies may apply to perception of real motion only to the extent to which real and apparent motion depend on the same underlying mechanisms (see Frisby, 1972; Wertheimer, 1912). 
The display consisted of an array of points that appeared to move horizontally on the face of an oscilloscope. Continuous motion was approximated by displacing each point successively by a small distance every $5 \mathrm{msec}$. Velocity was varied by the magnitude of displacement, and duration by the number of 5-msec steps. For example, for the points to move at a velocity of $10 \% \mathrm{sec}$, they were displaced $.05^{\circ}$ every $5 \mathrm{msec}$. Similarly, for a display to have a duration of $160 \mathrm{msec}$, the points were intensified a total of 33 times at 5-msec intervals. Amount of smear was estimated from observers' settings of a standard line to match the apparent length of the line segments produced by the moving points in the display.

This method of measuring extent of smear is homologous to a procedure commonly employed for estimating duration of visible persistence. For example, in an experiment by Efron and Lee (1971), a single rotating radial line was illuminated by a series of stroboscopic flashes, and the observers were required to report the number of lines that were visible simultaneously at any one time. Suppose that a flash occurred every $20 \mathrm{msec}$, and that the display remained in darkness between flashes. Suppose also that a hypothetical observer could achieve a representation of the display that was entirely veridical in all its spatiotemporal details. Under these conditions, the hypothetical observer would catch a quick glance of a radial line and another glance of it at the next radial location after a relatively long period of total darkness. Seen veridically, the display would remain dark for most of the time, except for glimpses of a single radial line illuminated briefly in a new location by each successive flash. Instead, what is seen is a group of radial lines moving together around a circular path. Many lines are seen at any one time, instead of just one, because of visible persistence. The number of lines seen simultaneously was utilized by Efron and Lee (1971) as a basis for estimating duration of visible persistence. Variants of this method have been commonly employed for the same purpose in investigations of visible persistence and its attenuation (e.g., Allport, 1968, 1970; Di Lollo \& Hogben, 1985; Dixon \& Hammond, 1972; Efron \& Lee, 1971; Farrell, 1984; Wade, 1974).

In the present work, as in Burr's (1980), only one point was ever physically present on the display surface at any one time, yet the perception was of many points-or lines-in vivid horizontal motion. The number of points seen simultaneously within a line was used here as the basis for estimating duration of visible persistence or, equivalently, duration of smear. In practice, the observer was required to set the length of a comparison line so that it matched that of the apparently moving lines. Details of how the observers' settings were employed to calculate duration of smear are given below in the description of Figure 3.

\section{Method}

Observers. The two authors served as observers in Experiment 1 Both had normal or corrected-to-normal vision.
Visual display. All stimuli were displayed on a HewlettPackard 1333A oscilloscope equipped with P15 phosphor. At the viewing distance of $57 \mathrm{~cm}$, one side of the $8 \times 8 \mathrm{~cm}$ display surface subtended a visual angle of $8^{\circ}$. The display consisted of an array of 100 points at random locations, each intensified for $1 \mu \mathrm{sec}$. To produce motion, the array was replotted with each point displaced a small horizontal distance from its previous position. The displacement was repeated as required to produce the desired combination of velocity and duration of motion.

Velocity was varied at three levels: $5.0,10.0$, and $15.0^{\circ} / \mathrm{sec}$ This was achieved by repeated displacement of the array by .25 , .50 , or $.75 \mathrm{~mm}$ on the display surface. Duration was varied at six levels: $10,20,30,40,80$, and $160 \mathrm{msec}$. This was achieved by displacing the array either $2,4,6,8,16$, or 32 times across the display surface. The direction of motion (either left or right) was chosen randomly on each trial.

Screen illumination was provided by a pair of shielded $15-\mathrm{cm}$ Sylvania F4T5CW fluorescent tubes located to the front and sides of the display surface, so as to produce an average luminance of $30 \mathrm{~cd} / \mathrm{m}^{2}$ as measured by a Spectra Spotmeter. The points were plotted at a constant intensity that made them comfortably visible under all conditions.

A horizontal standard line was displayed continuously in the center of the screen. In each condition, the separation between adjacent points in the standard line was the same as the distance between successive displacements in the array of moving points. The brightness of the standard line matched that of the array.

Procedure. Observers viewed the display binocularly from a distance of $57 \mathrm{~cm}$, set by a headrest. The standard line was shown continuously at the center of the screen, and served as an aid to fixation. The observer initiated a display of the moving array by pressing a button, and adjusted the number of points in the standard line so that its length matched that of the line segments produced by the moving points. The display could be repeated until the observer was satisfied with the adjustment. A total of 18 such adjustments were made within a session, one for each combination of velocity and duration. The 18 conditions were ordered randomly within a session. Each observer served in nine experimental sessions.

An equal number of control trials were run as a check on the measurement procedure. In the control trials, the 5-msec delay between successive displacements was eliminated so that all points were intensified effectively simultaneously. However, when this was done, the display appeared as an array of 100 stationary lines, many of which merged into each other. The overlap, which was particularly severe with the longer lines, prevented the observer from estimating the length of the individual lines. To avoid this, the number of points was reduced from 100 to 40 in the control trials.

To ensure that the different numbers of points in the experimental and control conditions had no effect, a subsidiary experiment was conducted. A complete set of data was obtained from 3 observers under the experimental conditions described above, except that the number of points in the array was systematically varied at four levels: $10,20,40$, and 80 . The number of points in the array had no effect.

\section{Results and Discussion}

Figure 1 shows the results of the control condition, separately for the two observers. The two lines in the graph represent the expected settings for veridical matching of the lengths of standard and comparison lines. The data points agree closely with veridical settings, with the exception of slight underestimates at the smallest interpoint separation, at which adjacent points could not be resolved. Overall, the results show that both observers could match standard and comparison stimuli with a high degree of accuracy. 


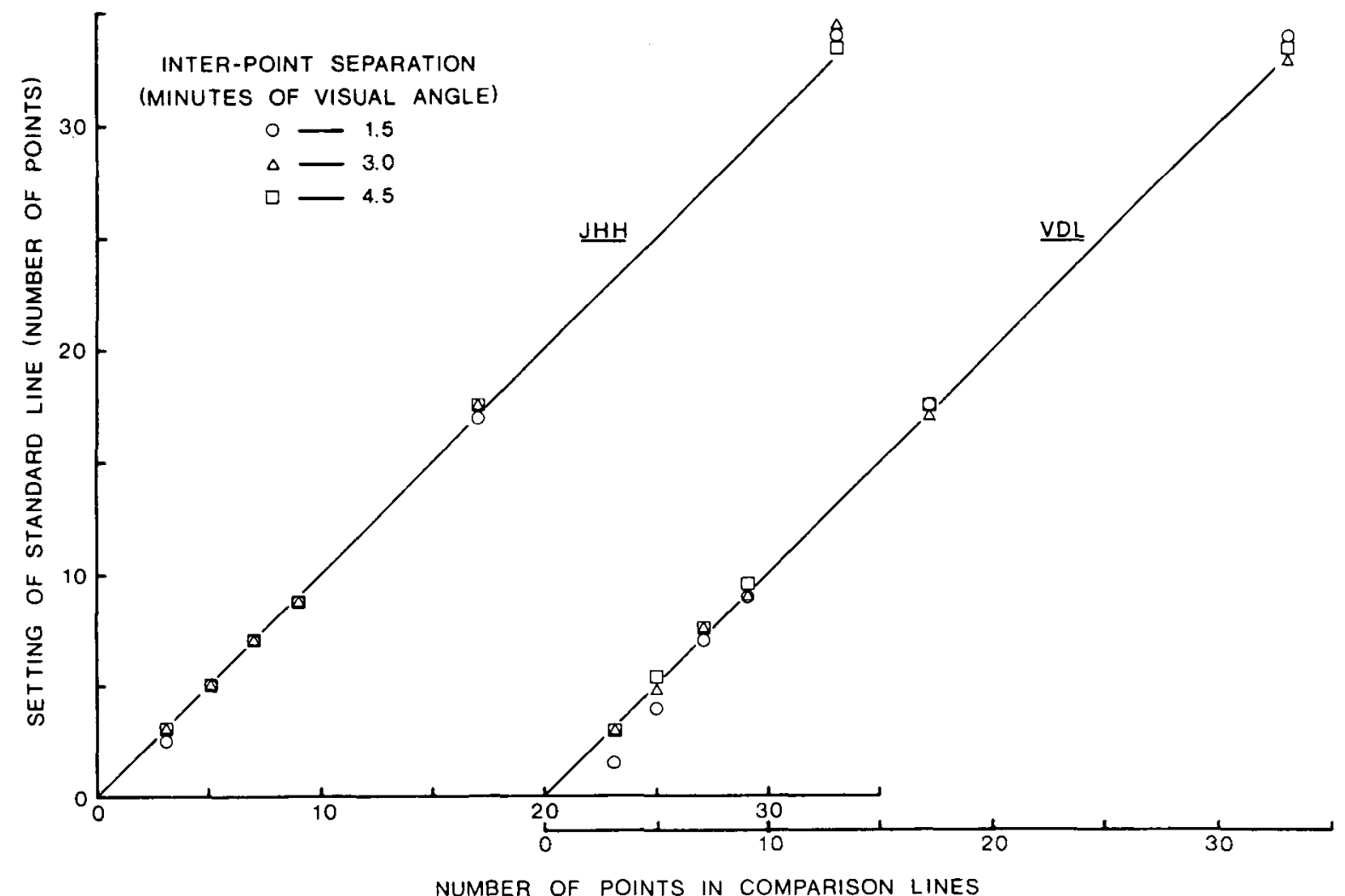

Figure 1. Number of points set in the standard line as a function of the number of points in the comparison line. Each datum represents the mean of nine settings.

Results for the experimental conditions are shown in Figure 2, plotted in the style followed by Burr (1980). Despite some differences, the overall pattern is not unlike that obtained by Burr. The most obvious difference is in the length of smear recorded. In Burr's study estimates never exceeded $10^{\prime}$ of arc, and were mostly below $5^{\prime}$. In the present work, estimates ranged up to $25^{\prime}$, and were below $5^{\prime}$ only for the lowest velocity. In addition, in the present work, estimates of apparent length of smear reached a maximum at longer durations of the display, and the decrement following the peak was not as pronounced as in Burr's study.

Phenomenologically, the most salient attribute of the display was a vivid appearance of motion seen at all but the shortest durations. The display appeared as an aggregate of horizontal lines in smooth coherent motion to the left or to the right, depending on the trial.

A limitation of presenting the data as we have in Figure 2 is that comparisons between results for different velocities are obscured by the confounding of velocity with extent of travel for a given duration of display. For example, at a duration of $10 \mathrm{msec}$, at which no attenuation of smear is expected, the extent of travel is $3^{\prime}$, $6^{\prime}$, or $9^{\prime}$ of arc for velocities of 5,10 , and $15^{\circ} / \mathrm{sec}$. Accurate setting of the standard line would thus differ depending on the velocity of the display, yet the three different settings would all represent complete absence of attenuation of smear. Similarly, attenuation of, say, $50 \%$ would be represented by different values on the ordinate of Figure 2, thus resulting in different scores for the same magnitude of effect. As a result, direct comparisons at any ordinate between velocity curves in Figure 2 will be misleading.

A representation of the data that permits direct comparison between velocity curves is shown in Figure 3. Here, the data have been rescored to reflect the temporal extent of smear rather than its apparent length on the screen. The scores in Figure 3 represent the duration of visible persistence required to maintain visibility of a moving segment whose length was specified by the observer's setting of the standard line. This method of estimating duration of smear is equivalent to a method of estimating duration of visible persistence employed in earlier studies (e.g., Efron \& Lee, 1971) and described in the introduction of the present study. In practice, this was done by subtracting 1 from the number of points in the standard line, and multiplying by $5 \mathrm{msec}$, rather than by the distance between points, as was done in Figure 2.

Expressing magnitude of smear in temporal units permits direct comparisons between velocities. It also permits the use of the same units of measurement for the assessment of smear and of visible persistence. What is more, it now becomes possible to draw a single line representing the theoretical setting of the standard line that 


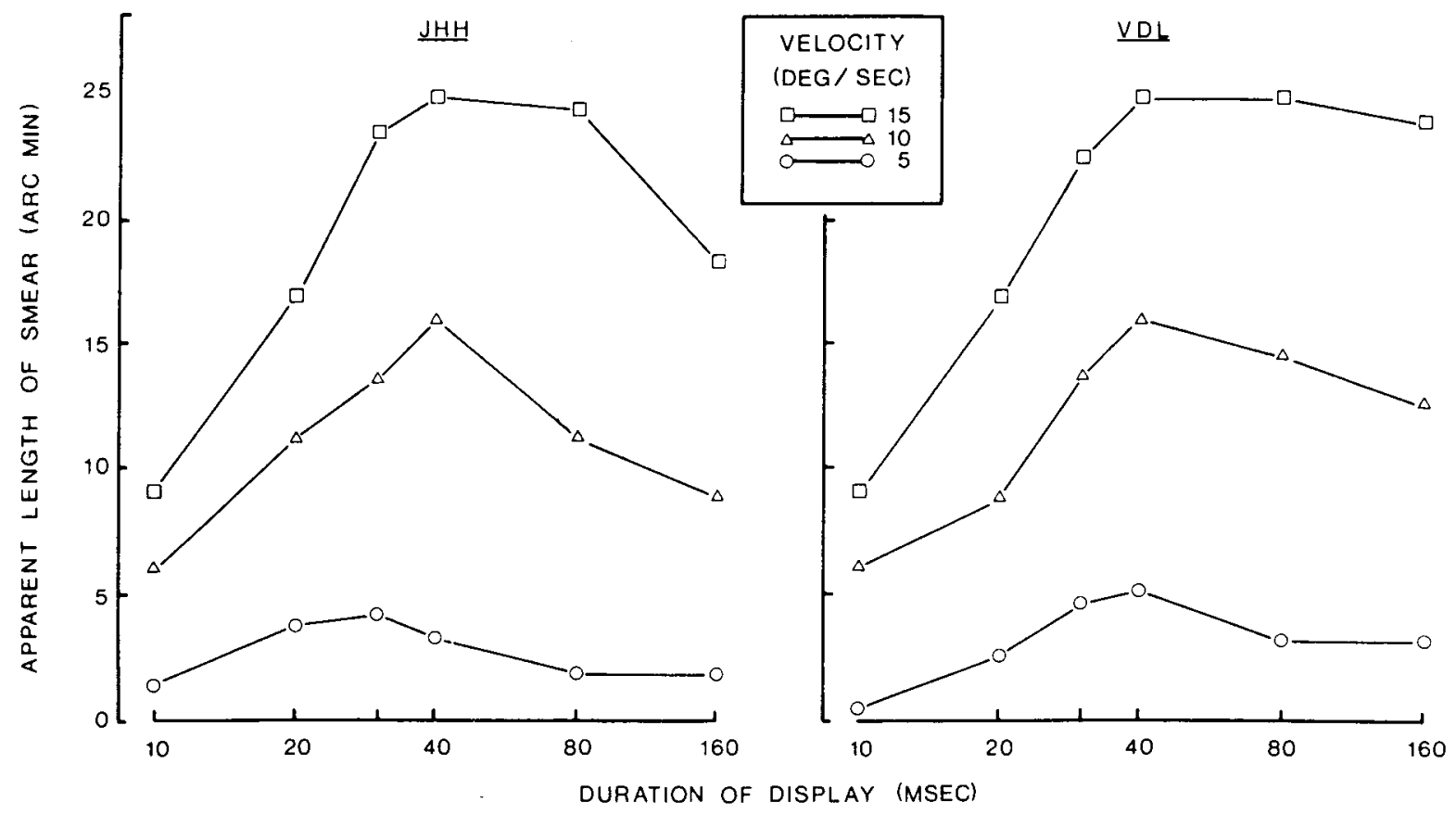

Figure 2. Apparent length of smear as a function of target velocity and duration of the display.

would be obtained with no attenuation of smear (see segmented line in Figure 3). The segmented line shows the duration of smear (or, equivalently, the duration of visible persistence) that would be obtained if visible persistence were at least as long as the duration of the display and there were no suppression. This line will be referred to as the "persistence line."

For both observers, duration of smear increases as duration of the display is increased to about $40 \mathrm{msec}$ and then falls off, reflecting increasing attenuation as the display is prolonged. Strong attenuation is evident even when smear is at a maximum: at a display duration of $40 \mathrm{msec}$, only a $27-\mathrm{msec}$ trail of smear is seen at the highest velocity, and even less at the lower velocities. With no attenuation, smear would have been $40 \mathrm{msec}$ in every case. The observed values represent attenuation of between $30 \%$ and $70 \%$, depending on velocity.

Velocity of the display affects duration of smear in two ways. First, the overall level of attenuation is greater at lower velocities. It must be noted that this result is independent of the actual length of smear seen on the screen. Whereas it is entirely to be expected that the length of a smear trail would be greater for objects moving at higher velocities (e.g., Burr, 1980), it is far from obvious that the duration for which smear persists within the visual system should depend upon the velocity of the stimulus.

Second, the rate at which duration of smear declines after peaking is greater at the lower velocities. As seen in Figure 3, attenuation of smear at the lowest velocity is virtually complete at a display duration of $80 \mathrm{msec}$. In contrast, there is a strong suggestion in the shape of the curves for the higher velocities that attenuation develops more gradually and that progressively more attenuation would be achieved at display durations beyond $160 \mathrm{msec}$.

A further aspect of the results, not apparent in Figure 2 but clearly evident in Figure 3, is the underestimation of smear at the lowest velocity when the duration of the display was brief. Under these conditions, judgments of length were difficult because the points making up the lines were few and close together, and the most salient subjective characteristic of the lines became brightness rather than length. As a result, there can be no confidence that judgments of length were uncontaminated by differences in brightness. This problem was even more severe when preliminary trials were run with a display velocity of $2.7 \% \mathrm{sec}$, as used by Burr (1980).

Even though Burr's (1980) results were not replicated in detail, the overall pattern is similar, and the technique has been shown to yield data that remain stable across observers. More importantly, the sharp deviation of all curves from the persistence line in Figure 3 points to some form of active process whose effect is to terminate visible persistence and reduce motion smear. We now turn to a consideration of this process.

An active process of suppression offers the simplest interpretation of the results; but first, an alternative hypothesis must be considered and rejected. It may be suggested that visible persistence remained uninhibited throughout its temporal course, but that its duration varied across experimental conditions. This hypothesis is unsatisfactory 


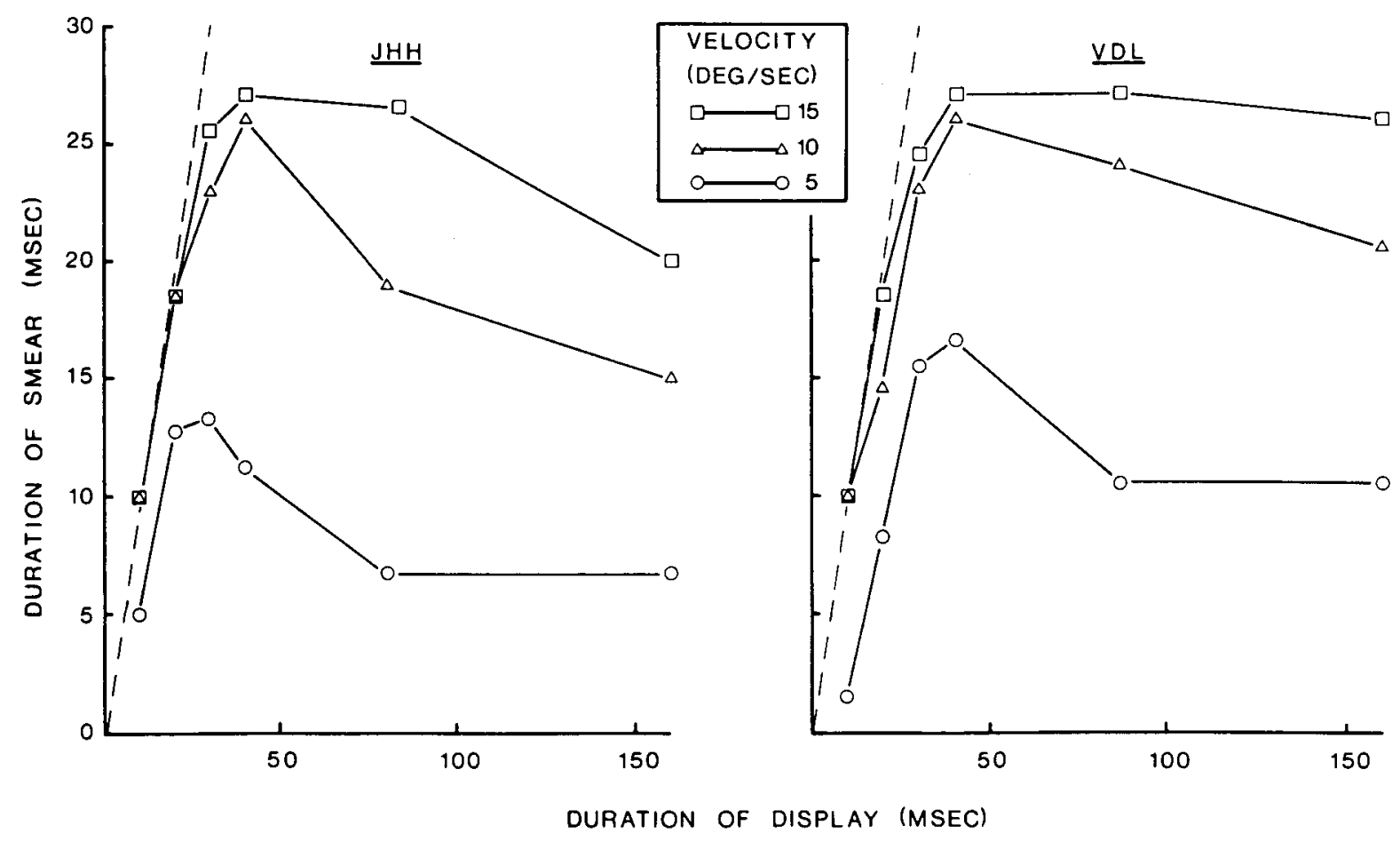

Figure 3. Duration of smear as a function of target velocity and duration of the display.

for two reasons. First, no duration of smear in Figure 3 exceeds $30 \mathrm{msec}$; this value is far below the values obtained in studies of visible persistence (e.g., Coltheart, 1980 ) and suggests the intervention of other factors. Second, if this hypothesis were true, duration of visible persistence would have to vary between about 5 and $30 \mathrm{msec}$, depending on velocity of the target, a dependence that would be ad hoc and implausible. On these grounds, it is unlikely that the measured duration of smear is merely reflecting different durations of visible persistence.

Suppression is a more credible alternative. It has been shown that strength of suppression increases with spatial proximity of successive points (Di Lollo \& Hogben, 1985; Dixon \& Hammond, 1972; Farrell, 1984). Proximity of points was employed in the present experiment to vary target velocity. The ostensible relation between target velocity and duration of smear (see Figure 3 ) is therefore explicable in terms of the dependence of suppression on proximity of points. Less smear was seen at lower velocities because successive points in these displays were closer together.

A likely mechanism underlying the effect of proximity may be found in some form of network inhibition at one or more levels in the visual system (Barlow, 1958; Ratliff, 1965; Singer \& Bedworth, 1973; Whitten \& Brown, 1973). A salient characteristic of network inhibition is that its strength depends on proximity of stimulated points. This characteristic is consistent with the suggestion that inhibition underlies the suppression of visible persistence. The role of inhibition as a basis for suppression is examined further in Experiment 2.

\section{EXPERIMENT 2}

\section{Method}

Observers. The two authors and a female student, who was experienced in similar psychophysical tasks but naive as to the purpose of the experiment, served as observers. All had normal or corrected-to-normal vision.

Visual displays. The visual displays were identical to those employed in Experiment 1, except that background illumination was varied as described below.

Procedure. The experimental conditions of Experiment 1 were replicated at three levels of background illumination and with varying levels of stimulus intensities. The levels of background illumination were $0.3,3$, and $30 \mathrm{~cd} / \mathrm{m}^{2}$, as measured by a Spectra Spotmeter.

At the lowest background illumination, five levels of stimulus intensity were employed. The lowest was chosen so that stimuli were barely but reliably visible against the background luminance of $0.3 \mathrm{~cd} / \mathrm{m}^{2}$. The remaining four were $.3, .6, .9$, and $1.12 \mathrm{log}$ units higher in intensity. The highest was the maximum possible on the oscilloscope.

At the intermediate background illumination $\left(3 \mathrm{~cd} / \mathrm{m}^{2}\right)$, only the four highest stimulus intensities were used because the lowest was not reliably visible. At the highest background illumination, only the two highest stimulus intensities were used, for the same reason.

Only one combination of stimulus intensity and background illumination was employed within a session. One session consisted of 18 adjustments of the standard line, one for each combination of six durations of the display $(10,20,30,40,80$, and $160 \mathrm{msec})$ and three velocities $(5,10$, and $15 \% \mathrm{sec})$. Each observer served in a total of 33 sessions, 3 at each combination of stimulus intensities and background illumination.

\section{Results and Discussion}

The phenomenological appearance of the displays in Experiment 2 was much the same as in Experiment 1. Nota- 

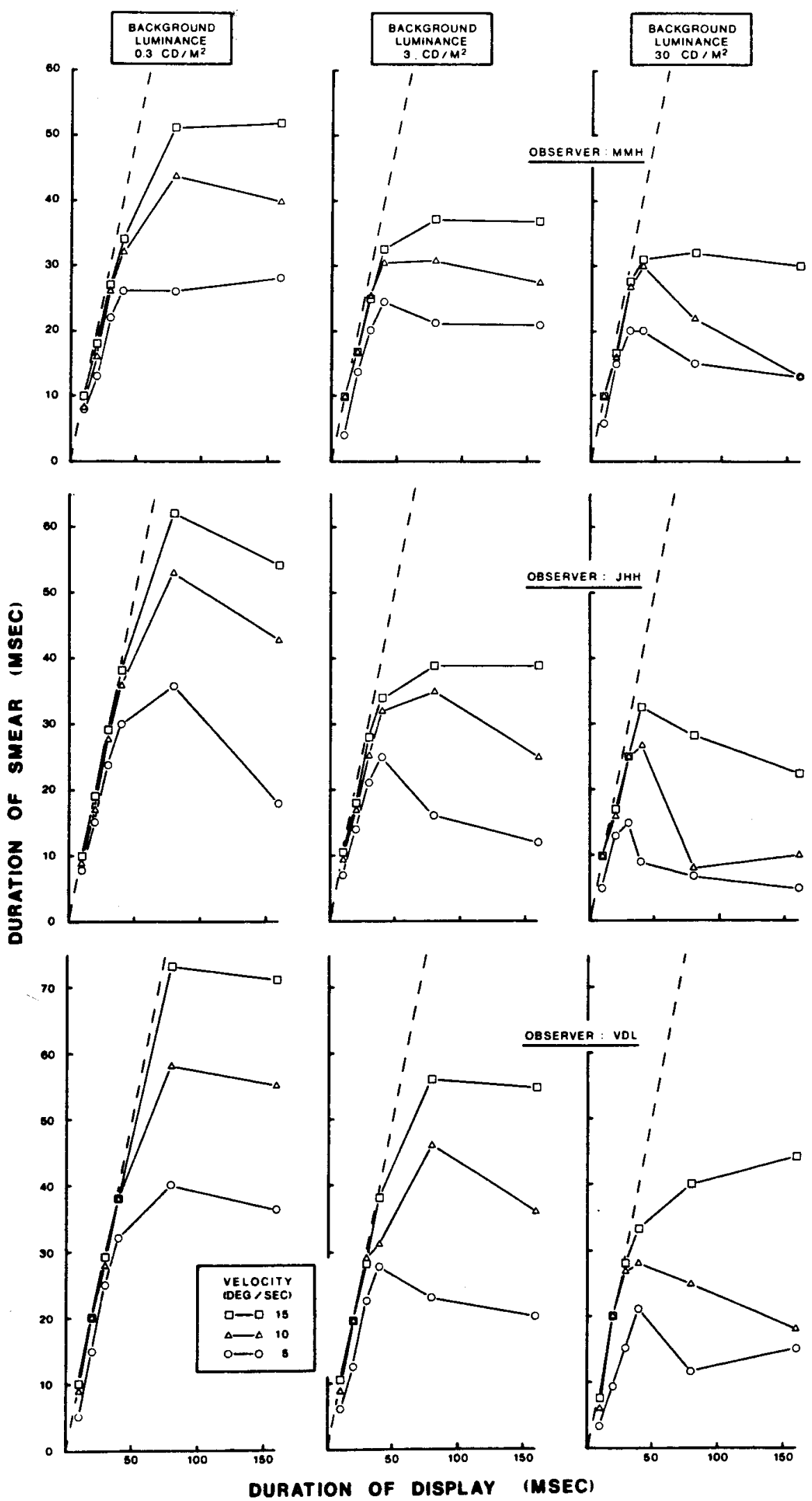

Figure 4. Duration of smear as a function of background luminance, target velocity, and duration of the display, separately for 3 observers. 
bly, the vivid appearance of coherent motion was maintained at all levels of background luminance and at all but the briefest durations of the display.

Although luminance of the background had a large effect on duration of smear (Figure 4), intensity of the stimulus had no effect at all. Take, for example, the condition with the lowest level of background luminance, in which the intensity of the stimuli was varied at five levels over a range of more than 1.0 log unit. Despite the large variation in stimulus intensity, the differences among the five means were negligible: averaged over the $3 \mathrm{ob}-$ servers, the mean duration of smear for the five stimulus intensities (from dimmest to brightest) were $30.0,28.7$, $29.1,30.0$, and $31.5 \mathrm{msec}$. Nor was there any evidence of interaction between stimulus intensity and other variables. Clearly, if stimulus intensity had any effect, it was too minor to warrant consideration in the present context. For economy and clarity of presentation, all data have been averaged over stimulus intensities in Figures 4 and 5. It is worth noting that the lack of an effect of stimulus intensity also means that contrast was not a salient determinant of the duration of smear.

The results of Experiment 2 are shown in Figure 4, separately for each observer. Within each panel, the results are similar to those of Experiment 1 . The similarity is greatest at a background luminance of $30 \mathrm{~cd} / \mathrm{m}^{2}$ (which matched that of Experiment 1), with progressively more smear being recorded at lower luminances. The overall effect of velocity was the same as in Experiment 1: Duration of smear increased with velocity at all levels of background luminance. As before, the effect is probably attributable to spatial proximity of successive points.

The main purpose of presenting the individual results, as in Figure 4, is to show the high degree of consistency among observers. The data in Figure 4 have been condensed and rearranged in Figure 5 so as to highlight the effect of background luminance, which was the variable of principal interest in the present experiment.

Background luminance can be seen to have two major effects. First, at longer display durations-where the curves lie well below the persistence line-degree of suppression is directly related to level of luminance. In each panel of Figure 5, the curves for the highest luminance show the least duration of smear. Second, luminance affects the time-course of the development of suppression: the higher the luminance, the shorter the stimulus duration at which suppression begins to appear. This can be seen in Figure 5 by noting the order in which the curves peel away from the persistence line: in each panel the curves peel away most rapidly when luminance is high and least rapidly when it is low. Correspondingly, suppression reaches an asymptotic level sooner at higher luminances. This can be seen best in the left panel of Figure 5, where at durations of 80 and $160 \mathrm{msec}$ the curve for the highest luminance is close to asymptotic, whereas that for the lowest luminance is still falling. Longer dis-

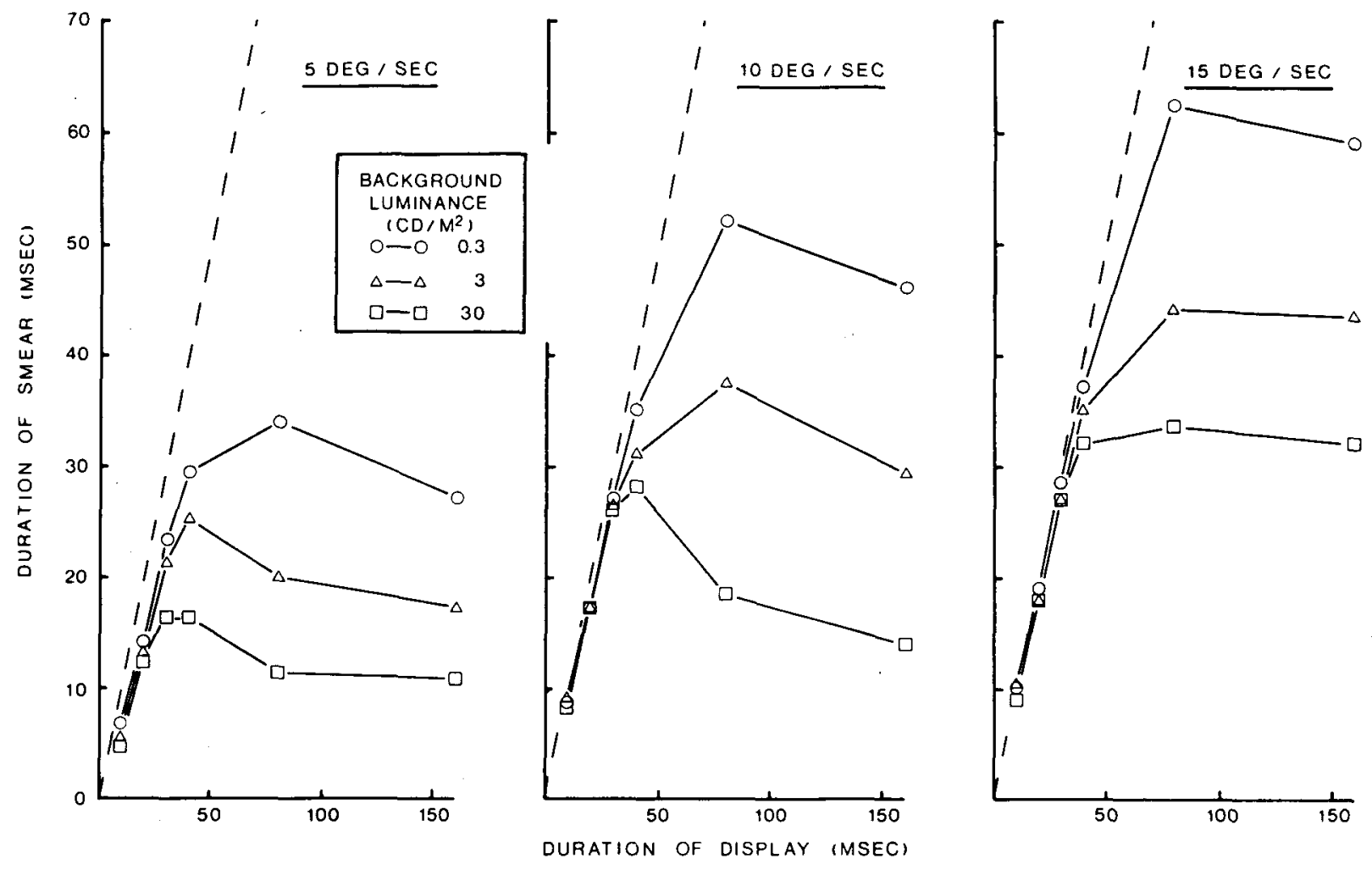

Figure 5. Duration of smear as a function of target velocity, background luminance, and duration of the display, averaged across observers. 
play durations would be required to establish the complete time-course of development of suppression, especially at lower background luminances.

The main findings of Experiment 2 can be summarized as follows: With higher luminance there is more suppression sooner.

\section{GENERAL DISCUSSION}

Besides the effect of display duration, which is discussed below, two variables were found to affect duration of smear in these experiments: velocity of the target and luminance of the background. Increments in velocity produced smear of longer duration; increments in luminance led to the twin effects of shorter duration of smear and earlier onset of its attenuation. We now consider alternative theoretical accounts of these findings.

According to Burr $(1980,1981)$, whether or not smear is produced by a moving stimulus depends on which of two sets of channels is activated by the display. Stationary or slowly moving stimuli activate a movementindependent system consisting of a set of channels tuned to relatively high spatial and low temporal frequencies. By contrast, rapidly moving stimuli activate a movement system consisting of a different set of channels, tuned to relatively low spatial and high temporal frequencies. The movement system is said to summate the energy from successive positions of a moving stimulus and to be free from smear. Motion smear results from inappropriate activation of the movement-independent system by a moving stimulus. Smear is avoided when the movement system is engaged. Presumably, the movement system has the longer latency, so that at the very beginning of a motion sequence only the movement-independent system is engaged, producing smear. After a delay, the movement system takes over and smear is avoided.

How does this model account for the finding that duration of smear is less with slow-moving targets? If smear is avoided by engaging the movement system, it is difficult to see why a slow-moving stimulus should be more effective than a fast-moving one in engaging a system ostensibly attuned to the perception of moving objects. Nor can it be said that the slowest stimuli in our displays failed to engage the movement system. Had this been the case, the displays would have engaged only the movementindependent system and should have produced smear of longer-not shorter-duration. Figure 3 shows that, even at the lowest velocity, duration of smear first increased and then decreased as a function of stimulus duration, clearly suggesting some form of attenuation. It is this evidence of attenuation that is in need of explanation.

Similar difficulties are encountered by this model in explaining the effects of background luminance. The finding that less smear is obtained at higher luminances would suggest that light potentiates the low-spatial-frequency movement system. This is an intriguing assumption that may be worth pursuing, but that must be regarded as entirely ad hoc until justified on independent grounds. As for the finding that attenuation of smear occurs more rapidly at higher background luminance, the model must resort to a similarly ad hoc assumption that the latency of the movement system is inversely related to luminous intensity.

It must be stressed that these difficulties do not invalidate Burr's $(1980,1981)$ treatment of the temporal summation of moving images. But the model is clearly in need of elaboration if it is to account for the effects of velocity and luminance on the reduction of motion smear.

Another interpretation of our data can be couched in terms of a filtering model that was initially developed to account for the "interpolation effect"' (Morgan, 1976; Morgan \& Watt, 1983), but that has implications for the perception of motion smear (M. J. Morgan, personal communication, June 1984). The interpolation effect is obtained in a vernier acuity task, with targets apparently moving through successive stations. Under appropriate spatiotemporal conditions, observers perceive the position of the bars as interpolated between stations.

As applied to motion smear, the crucial aspect of the model consists of a pair of filters, one temporal and the other spatial. The temporal filter causes stimuli at successive stations to persist for a period determined by the temporal constant of the filter. The output of the temporal filter is then passed through a spatial filter whose constant is determined by a difference-of-Gaussian (DOG) function. As long as the output of the DOG filter does not contain more than one zero crossing, only one stimulus is seen, regardless of the number of stimuli that entered the filter at the same time. Within this conceptual framework, absence of motion smear is regarded as a failure to resolve separate stimuli within the spatial filter. That is, even though several points enter the spatial filter, only one point is seen. On the other hand, presence of smear indicates that the separation between points is sufficient to ensure their resolution. Smear is perceived in the form of multiple stimuli.

The interpolation model accounts well for the finding that duration of smear is greater at higher velocities. As noted above, velocity was manipulated by varying the spatial separation between successive points. At wide interpoint separations (i.e., at high velocities), successive points matched or exceeded the spatial domain at the filter, thereby becoming resolvable as distinct points which were seen as smear (Morgan \& Watt, 1983).

Background luminance is handled less directly. The finding that duration of smear is negatively related to background luminance could be handled within the interpolation model by assuming that the constant of the temporal filter decreases as luminance is increased. This would lead to fewer points (i.e., less smear) being perceived simultaneously at higher levels of luminance. In accord with this assumption, there are clear indications in the experimental literature that increments in luminance should reduce the constant of the temporal filter. Roufs and Blommaert (1981), from whom Morgan and Watt (1983) obtained an estimate of the temporal constant, have shown 
that the temporal impulse function has far greater spread with low than with high background luminance. Kelly (1971) reported the same relationship. In the same vein, it could be assumed that increments in luminance may reduce the constant of the spatial filter, perhaps through expanded inhibitory side-bands within a receptive field, thus reducing extent of smear. However, in its present form, the model has no means of accounting for the finding that attenuation of smear occurs more rapidly at higher background luminance. Morgan and Watt (1982) did discuss the effect of duration on the interpolation effect, but the explanations they considered lie outside the scope of the interpolation model.

In sum, although the interpolation model was developed to account for the illusory spatiotemporal alignment of elements in a stimulus train, rather than for smear per se, it seems capable of accounting for the present results with only a few plausible additional assumptions. In this respect, it would be of interest to find out how the interpolation effect itself varies as a function of background luminance.

A third explanation of our results can be given in terms of inhibitory interactions between transient and sustained activity produced in the visual system by spatially adjacent stimuli displayed in rapid sequence. This approach, first proposed and developed by Breitmeyer (1975a, 1975b; Breitmeyer, Battaglia, \& Weber, 1976; Breitmeyer \& Ganz, 1976; Breitmeyer, Love, \& Wepman, 1974), has been employed successfully as an explanatory basis of contour suppression in metacontrast as well as in apparent motion (e.g., Hogben \& Di Lollo, 1984; von Grünau, 1981). On the strength of psychophysical and neurophysiological evidence, it is assumed that stimulation activates two sets of visual pathways, or channels, having distinct spatiotemporal response characteristics. The transient channel has short latency and responds optimally to the low spatial frequencies of fast-changing stimuli; by contrast, the sustained channel has longer latency and is attuned to processing figural aspects of the stimulus such as details carried by the higher spatial frequencies. Activity in the transient channel is regarded as having an inhibitory effect on concurrent activity in the sustained channel.

Perceptual suppression of the contours of a temporally leading stimulus is explained in terms of the joint effects of the shorter latency of transient responses and of transient-on-sustained inhibition. Given two spatially adjacent stimuli displayed in succession at the appropriate temporal interval, the fast transient activity produced by the temporally trailing stimulus will inhibit the ongoing more sluggish sustained activity produced by the leading stimulus, thus suppressing perception of its contours and of related figural details.

We wish to stress that this is only one of several models that favor inhibition as the basis for suppression of contour information in metacontrast and in apparent motion (e.g., Matin, 1975; Weisstein, Ozog, \& Szoc, 1975). It is not our aim to review each individual model; suffice it to say that, although they differ in detail, inhibition- based models can provide an adequate account of the pattern of results obtained in the present work.

Within this general conceptual framework, we suggest that suppression of visible persistence-or attenuation of smear-is based on inhibitory processes that may be diverse in character and occur at more than one level within the visual system. Besides such classic studies of retinal inhibition as those of Barlow, Fitzhugh, and Kuffler (1957) and Ratliff (1965), the work of Whitten and Brown (1973) reveals suppression of rod activity by lateral inhibition from the cones; at the level of the lateral geniculate nucleus, Singer and Bedworth (1973) found that activation of $Y$ cells in the cat inhibits ongoing activity of $\mathrm{X}$ cells. Although at this stage it is not possible to be specific as to either locus or mechanism, it is clear that inhibition provides a plausible basis for suppression of visible persistence. Three characteristics of inhibition are of particular relevance to the present work: inhibition decreases with distance between stimulated points, it increases with background illumination or light adaptation, and it requires some time to develop (e.g., Barlow et al., 1957; Ratliff, 1965; Whitten \& Brown, 1973). Below, we examine the relation of these characteristics of inhibition to the present findings.

At issue in the present work is the effect of luminance of the display on duration of smear. Although the intensity of the points had no effect, luminance of the background was a powerful determinant of smear: as background luminance increased, duration of smear decreased. This finding is precisely what would be expected if reduction of smear resulted from a process of suppression based on inhibitory activity. A direct relationship between background luminance and level of inhibition has been confirmed both neurophysiologically and psychophysically. From single-unit recordings in the cat's visual system, Barlow et al. (1957) found that inhibitory activity was high at photopic levels but totally absent in dark adaptation. Similarly, psychophysical studies by Ikeda (1965) showed that inhibition becomes progressively weaker as the adapting luminance is lowered. In the suppression hypothesis, the stronger inhibition associated with higher luminance acts to terminate visible persistence, reducing the duration of smear.

Velocity of the target affected duration of smear about as powerfully as background luminance: increments in velocity increased the duration of smear. As noted above, in our studies as well as in Burr's (1980), the spatial separation between successive points increased with velocity. In the present context, increased suppression of smear at lower velocity is attributable to the stronger inhibition that is obtained at shorter interpoint separations. It is well known that strength of inhibitory interactions, at least at the retinal level, increases with proximity of stimuli (e.g., Ratliff, 1965). Psychophysical evidence consistent with this thesis has been reported by Allport (1968). Di Lollo and Hogben (1985), Dixon and Hammond (1972), and Farrell (1984), who found that duration of visible persistence increases with separation between successive elements in a display. Given these premises, the obtained 
direct relationship between velocity and smear would follow.

Even though this account of the effect of target velocity is plausible, we must consider the possibility that the fundamental variable underlying the effect may be not interpoint separation but velocity per se, which would be handled within the visual system by separate channels tuned to different velocities. Although this is a possibility, the relation between activity in the separate velocitytuned channels and the duration of smear would still be in need of explanation.

Inhibition can also account for the puzzling finding that duration of smear first increased and then decreased as stimulus duration was increased. Up to stimulus durations of 30-40 msec, duration of smear followed the persistence line, showing no evidence of suppression (Figure 3). Thereafter, suppression developed rapidly, causing the curves to deviate sharply from the persistence line. This result is consistent with Barlow et al.'s (1957) finding that inhibition develops more slowly than excitation; they postulated a recruitment period that would be necessary for inhibition to reach full strength. Thus, brief stimuli (30-40 msec under our conditions) are not affected by inhibition and exhibit smear in degrees compatible with unsuppressed visible persistence. Furthermore, our finding that the curves peel off from the persistence line more rapidly at higher luminance (Figure 5) is consistent with the finding that bright backgrounds yield shorter critical durations for Bloch's law (Ganz, 1975). The shorter critical durations have been regarded (e.g., by Barlow, 1958) as deriving from an accentuation of inhibition at higher background intensities.

Indeed, the question may be raised as to whether the notion of inhibition is necessary to explain why increments in background luminance produced shorter durations of smear (Figure 5). It is well known that duration of visible persistence of static stimuli is less at higher photopic levels (Coltheart, 1980); put differently, it is known that the visual system shows less static summation as light adaptation level increases (see Ganz, 1975, for a review). It may be suggested that, although suppression can hardly be denied as a determinant of the present findings, the specific result showing reduction in motion smear with increased background intensity might indicate a change not in the level of suppression, but only in the period of summation.

Upon consideration, the two alternatives turn out to be homologous and probably related as cause and effect. There are strong psychophysical and physiological suggestions that inhibitory processes provide the basis for reducing the critical period of temporal summation. In a series of psychophysical studies of temporal summation with double pulses, Ikeda (1965) obtained clear evidence that the strength of inhibitory interactions between two pulses increased with background luminance, thereby diminishing the period of summation. Even more telling, in this respect, are the findings of Whitten and Brown
(1973), who studied inhibitory network interactions between cones and rods in the macaque monkey. They reported that inhibition of rods by cones greatly reduced the period of temporal summation as light adaptation increased. They went on to suggest that "the cone-rod lateral inhibitory pathway may free the cone system from interference by very slowly decaying rod signals at photopic intensities, thereby permitting the cone system to realize its full capability for resolving stimuli in the time domain" (Whitten \& Brown, 1973, p. 1652). A similar relation between summation and inhibition was pointed out by Barlow (1958), who noted that "the conditions... which diminish spatial summation in human experiments are the conditions which accentuate lateral inhibition in animal experiments" (Barlow, 1958, p. 348).

In essence, we suggest that the effect of background luminance illustrated in Figure 5 may be viewed just as appropriately in terms of summation as in terms of inhibition. Whether one or the other concept is employed will depend on the broader theoretical context and on the level of explanation required within that context.

\section{REFERENCES}

AllPort, D. A. (1968). Phenomenal simultaneity and the perceptual moment hypothesis. British Journal of Psychology, 59, 395-406.

Allport, D. A. (1970). Temporal summation and phenomenal simultaneity: Experiments with the radius display. Quarterly Journal of Experimental Psychology, 22, 686-701.

BARLOw, H. B. (1958). Temporal and spatial summation in human vision at different background intensities. Journal of Physiology, 141, 337-350.

Barlow, H. B., Fitzhugh, R., \& Kuffler, S. W. (1957). Change of organization in the receptive fields of the cat's retina during dark adaptation. Journal of Physiology, 137, 338-354.

Breitmeyer, B. (1975a). Predictions of U-shaped visual backward pattern masking from considerations of the spatio-temporal frequency response. Perception, 4, 297-304.

Breitmeyer, B. (1975b). Simple reaction time as a measure of the temporal response properties of transient and sustained channels. Vision Research, 15, 1411-1412.

Breitmeyer, B. G., Battaglia, F., \& Weber, C. (1976). U-shaped backward contour masking during stroboscopic motion. Journal of Experimental Psychology: Human Perception \& Performance, 2, 167-173.

BREITMEYER, B., \& GANZ, L. (1976). Implications of sustained and transient channels for theories of visual pattern masking, saccadic suppression, and information processing. Psychological Review, 83, 1-36.

Breitmeyer, B. G., Love, R., \& Wepman, B. (1974). Contour suppression during stroboscopic motion and metacontrast. Vision Research, 14, 1451-1456.

BurR, D. (1980). Motion smear. Nature, 284, 164-165.

BurR, D. C. (1981). Temporal summation of moving images by the human visual system. Proceedings of the Royal Society, London, B, 211, 321-339.

Coltheart, M. (1980). Iconic memory and visible persistence. Perception \& Psychophysics, 27, 183-228.

Di Lollo, V., \& Hogben, J. H. (1985). Suppression of visible persistence. Journal of Experimental Psychology: Human Perception \& Performance, 11, 304-316.

DiXoN, N. F., \& HAMMOND, J. (1972). The attenuation of visual persistence. British Journal of Psychology, 63, 243-254.

EFron, R., \& LEE, D. N. (1971). The visual persistence of a moving stroboscopically illuminated object. American Journal of Psychology, 84, 365-375. 
FARRELL, J. M. (1984). Visible persistence of moving objects. Journal of Experimental Psychology: Human Perception \& Performance, 10 , 502-511.

FrisBy, J. P. (1972). The effect of stimulus orientation on the phi phenomenon. Vision Research, 12, 1145-1166.

GANZ, L. (1975). Temporal factors in visual perception. In E. C. Carterette \& M. P. Friedman (Eds.), Handbook of perception: Vol. V. Seeing. New York: Academic Press.

Hogben, J. H., \& Di Lollo, V. (1984). Practice reduces suppression in metacontrast and in apparent motion. Perception \& Psychophysics, $35,441-445$.

IKEDA, M. (1965). Temporal summation of positive and negative flashes in the visual system. Journal of the Optical Society of America, 55, 1527-1534.

KeLly, D. H. (1971). Theory of flicker and transient responses: I. Uniform fields. Journal of the Optical Society of America, 61, 537-546.

Matin, E. (1975). The two-transient (masking) paradigm. Psychological Review, 82, 451-461.

Morgan, M. J. (1976). Pulfrich effect and the filling in of apparent motion. Perception, 5, 187-195.

Morgan, M. J., \& WATt, R. J. (1982). Effect of motion sweep duration and number of stations upon interpolation in discontinuous motion. Vision Research, 22, 1277-1284.

MorGan, M. J., \& WATT, R. J. (1983). On the failure of spatiotemporal interpolation: A filtering model. Vision Research, 23, 997-1004.
RatLifF, F. (1965). Mach bands: Quantitative studies on neural networks in the retina. New York: Holden-Day.

Roufs, A. J., \& BlommaerT, F. J. J. (1981). Temporal impulse and step responses of the human eye obtained psychophysically by means of drift-correcting perturbation technique. Vision Research, 21, 1203-1221.

SINGER, W., \& BEDWORTH, N. (1973). Inhibitory interaction between $\mathrm{X}$ and $\mathrm{Y}$ units in the cat lateral geniculate nucleus. Brain Research, 49, 291-307.

voN GrünaU, M. W. (1981). The origin of pattern information of an apparently moving object during stroboscopic motion. Perception \& Psychophysics, 30, 357-361.

WADE, N. J. (1974). Some perceptual effects generated by rotating gratings. Perception, 3, 169-184.

Weisstein, N., Ozog, G., \& Szoc, R. (1975). A comparison and elaboration of two models of metacontrast. Psychological Review, 82, 325-343.

Wertheimer, M. (1912). Experimentelle Studien über das Sehen von Bevegung. Zeitschrift für Psychologie, 61, 161-265.

WhitTen, D. N., \& Brown, K. T. (1973). Photopic suppression of monkey's rod receptor potential, apparently by cone-initiated lateral inhibition. Vision Research, 13, 1629-1658.

(Manuscript received May 29, 1985; revision accepted for publication October 18, 1985.) 